\title{
TCP with Network Coding - A Novel Scheme to Improve Performance in Wireless Multihop Networks
}

\author{
Sunil Bajeja \\ Asst. Prof. - FCA \\ Marwadi Edu. Fou. Grp of Inst.
}

Rajkot

\author{
Atul Gonsai, PhD \\ Associate Prof. - CSD \\ Saurashtra University \\ Rajkot
}

\begin{abstract}
Network coding is a way of improving performance in wireless networks by combining two or more packets to send together as a single packet. Two or more packets are combined together to form a single packet. the main goal of using network coding is to reduce bandwidth requirement. Wireless networks often suffer from various issues like collision, transmission errors etc due to broadcasting of signals. Network coding helps in reducing such issues and improving performance significantly. Various network coding schemes are implemented at physical layer, data link layer and network layer. This research paper introduces a novel network coding scheme TCP-NC - TCP with Network Coding at transport layer. TCP's most widely deployed variant - TCP NewReno is used. Simulation is performed in NS 2.35. Scenario based wireless multihop networks are designed with different scenarios based on number of nodes, number of hopes and type of network(poor or congested). TCP-NC shows a significant amount of improvement when used with the poor network.
\end{abstract}

\section{General Terms}

Networks, Network Packet transfer technique

\section{Keywords}

Network Coding, TCP, New Reno, Throughput, Packet Delivery Ratio

\section{INTRODUCTION}

Network coding is about encoding more than packets to form a single packet. Network coding is used to reduce number of transmissions to reduce requirement of bandwidth. Wireless networks often suffer from issues like collision, transmission errors, hidden and exposed station problems etc. Various network coding schemes are implemented at physical layer, data link layer and network layer. This research paper introduces a novel network coding scheme TCP-NC - TCP with Network Coding at transport layer. TCP's most widely deployed variant - TCP NewReno is used. Simulation is performed in NS 2.35. Scenario based wireless multihop networks are designed with different scenarios based on number of nodes, number of hopes and type of network (poor or congested). TCP-NC shows a significant amount of improvement when used with the poor network.

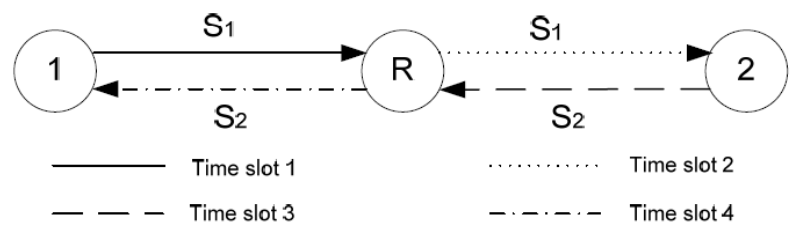

Figure 1. Network without Network Coding
Here node_1 and node_2 wants to exchange two messages S1 and S2 respectively. The topology has one intermediate node_R. in 1st step, node_1 will send S1 to node_R. in 2nd step, node_R will send S1 to node_2. In 3rd step, node_2 will send S2 to node_R and in 4th step, node_R will send S2 to node_1. In this way, in four steps of transmissions both the messages are exchanged with each other. Our goal is to reduce this requirement of four steps to reduce total number of transmissions. The following sections show various methods of coding packets [1][2][3].

\section{A SIMPLE NETWORK CODING}

Here node_1 and node_2 wants to exchange two messages S1 and S2 respectively. The topology has one intermediate node_R. in 1st step, node_1 will send S1 to node_R. in 2nd step, node_2 will send S2 to node_R. In 3rd step, node_R will combine S1 and S2 and will send combined message SR to node_1 and node_2 by broadcasting. In 4th step, node_1 will recover S2 and node_2 will recover S1 as shown below[1][2][3][4].

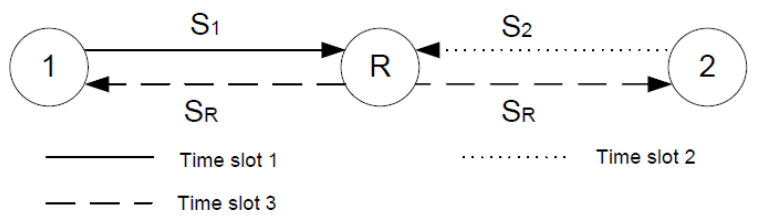

Figure 2. Straight forward Network Coding

Node $\mathrm{R}$ broadcasts $\mathrm{SR}=\mathrm{S} 1 \oplus \mathrm{S} 2$ where $\oplus$ is $\mathrm{XOR}$

Node 1 has $S 1$ and so it decodes

$$
\mathrm{S} 2=\mathrm{S} 1 \oplus \mathrm{SR}=\mathrm{S} 1 \oplus(\mathrm{S} 1 \oplus \mathrm{S} 2)
$$

Node 2 has S2 and so it decodes

$$
\mathrm{S} 1=\mathrm{S} 2 \oplus \mathrm{SR}=\mathrm{S} 2 \bigoplus(\mathrm{S} 1 \oplus \mathrm{S} 2)
$$

Let's say S1 $=10110$ and S2 $=11011$

$$
\mathrm{SR}=\mathrm{S} 1 \oplus \mathrm{S} 2=10110 \oplus 11011=01101
$$

$\mathrm{S} 2$ can be retrieved at Node 1

$$
\mathrm{S} 2=\mathrm{S} 1 \oplus \mathrm{SR}=10110 \oplus 01101=11011
$$

S1 can be retrieved at Node 2

$$
\mathrm{S} 1=\mathrm{S} 2 \oplus \mathrm{SR}=11011 \oplus 01101=10110
$$

As a part of literature survey, researcher has also referred various schemes which are compared below. 
Table 1. Various Network Coding Schemes[5][6][7][8][9][10][11][12]

\begin{tabular}{|c|c|c|c|c|c|}
\hline Scheme & $\begin{array}{c}\text { Methodolog } \\
\mathbf{y}\end{array}$ & $\begin{array}{c}\text { Applicati } \\
\text { on }\end{array}$ & Type & $\begin{array}{c}\text { Catego } \\
\text { ry }\end{array}$ & Links \\
\hline COPE & Inter-session & Unicast & XOR & Local & Lossy \\
\hline MORE & Intra-session & unicast & RL & Global & Lossy \\
\hline MORE & Intra-session & Multicast & RL & Global & Lossy \\
\hline Pacifier & Intra-session & Multicast & RL & Global & Lossy \\
\hline CODEB & Inter-session & $\begin{array}{c}\text { Broadcas } \\
\mathrm{t}\end{array}$ & XOR & Local & Perfect \\
\hline Dir. Ant. & Inter-session & $\begin{array}{c}\text { Broadcas } \\
\mathrm{t}\end{array}$ & XOR & Local & Perfect \\
\hline NAK & Intra-session & $\begin{array}{c}\text { Broadcas } \\
\mathrm{t}\end{array}$ & RL & Global & Lossy \\
\hline
\end{tabular}

\section{PROPOSED ALGORITHM}

Most of the Network coding techniques are implemented at physical layer, data link layer or transport layer. A very few techniques like COPE are implemented at transport layer. Transmission Control Protocol is connection oriented and reliable protocol which provides process to process communication. TCP is most widely used protocol in wired as well as wireless networks at transport layer. Wireless communication has its own limitations and disadvantages because of vulnerabilities of wireless mediums and broadcast nature. In such scenario, it is highly required to develop a system which deals which poor network and related issues. A poor network can be defined as having transmission impairments related issues like noise, distortion, cross talk, interference. Such network causes packet drops or packet corruption at a significant rate. TCP has no inherent capability to deal with wireless transmission issues. At the same time, all the network coding schemes which are proposed so far is expensive. In this thesis, researcher tried to incorporate network coding with TCP. The main goal is to enable network coding selectively. Our proposed protocol TCPNC - "TCP with Network Coding" becomes active based on prediction of reliability .TCPNC tries to improve performance in poor network by eliminating need of retransmissions by using network coded packets to recover lost / corrupted packets. At the same time, existing TCP works as it is.To test the TCPNC with existing TCP NewReno which has no network coding, researcher has used scenario based Wireless Multihop Networks. These networks are of different number of nodes where FTP traffic is scheduled for 5 minutes between a pair of nodes connected via multiple hopes. Error rates and noise are used to make the network poor. Queue length is used to make the network congested.

\subsection{Sender Side Pseudo code On_Packet_Sent( ) \\ \{}

Packets_Sent ++

If Sent Packet was Retransmitted Then

Packets_Retransmitted ++

End If

Reliability $=($ Packets_Sent -

Packets_Retransmitted) / Packets_Sent
\{

Send Packet $\mathrm{P}_{\mathrm{i}}$

Send Packet $\mathrm{P}_{\mathrm{i}+1}$

If Reliability $<2 / 3$ Then

$\mathrm{P}_{\mathrm{NW}}=\mathrm{P}_{\mathrm{i}} \oplus \mathrm{P}_{\mathrm{i}+1} \quad$ Send Redundant Network Coded Packet

TCP Header

Mark This Packet as a Network Coding in

End If

\}

TCPNC follows the phases of TCP NewReno. In additional of those phases, TCPNC keeps information about number of sent packets and number of retransmitted packets. After sending a packet, reliability is calculated based on the ratio of sent and retransmitted packets. If reliability is less than $2 / 3$, it is predicted that network is less reliable, here network coding is activated. TCPNC, XORes most recent two packets to form network coded packet $\mathrm{PNW}=\mathrm{Pi} \oplus \mathrm{Pi}+1$.After sending network coded packet, TCPNC continues just like TCP NewReno. The same can be shown in the form of flow chart in Figure 3.

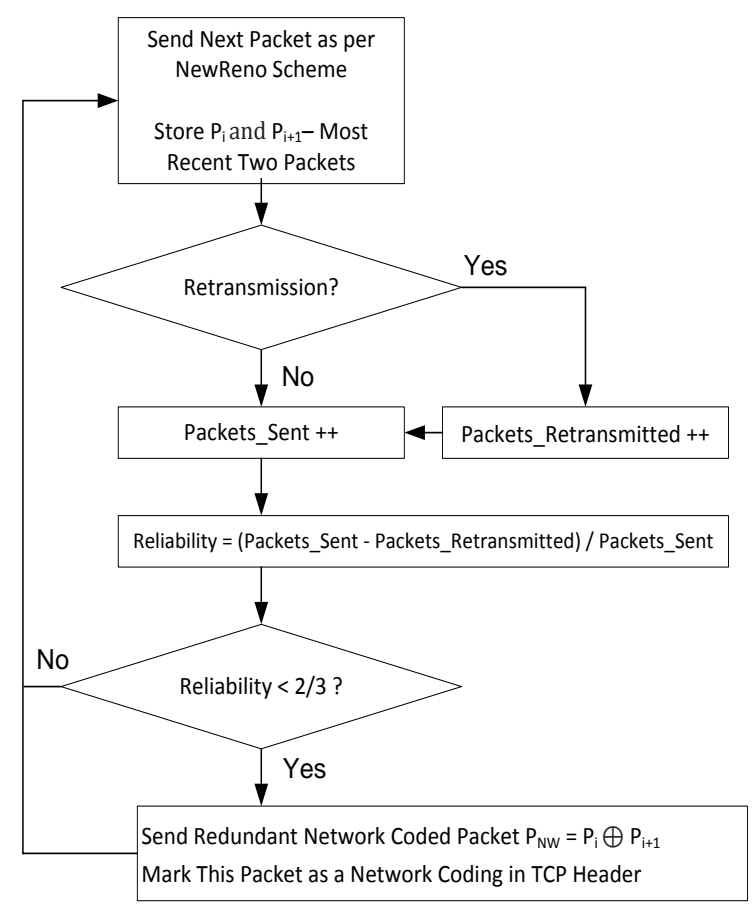

Figure 3. - Sender Side Flowchart

\subsection{Receiver Side Pseudocode} Receive_Packet ( )

\{

Try to Receive Packet $P_{i}$

Try to Receive Packet $\mathrm{P}_{\mathrm{i}+1}$

Try to Receive Packet $\mathrm{P}_{\mathrm{NW}}$

If $\mathrm{P}_{\mathrm{i}}$ was lost or corrupted then retrieve it $\mathrm{P}_{\mathrm{i}}=\mathrm{P}_{\mathrm{i}+1} \oplus \mathrm{P}_{\mathrm{NW}}$ If $\mathrm{P}_{\mathrm{i}+1}$ was lost or corrupted then retrieve it $\mathrm{P}_{\mathrm{i}+1}=\mathrm{P}_{\mathrm{i}} \oplus \mathrm{P}_{\mathrm{NW}}$

Next_Packets ( ) 
TCPNC tries to recover packets using network coding if any of the packets are lost or corrupted. TCP Receiver continuously receives packets. It checks whether a packet is network coded by checking its header. If a packet is network coded, TCPNC tries to find out whether previous two packets are properly received or not. If previous two packets were properly received then TCPNC simply discards network coded packet as it is no use of. If any of the previous two packets were lost, TCPNC uses network coded and most recently received packet to retrieve lost or corrupted packet as shown in flowchart. The same can be shown in the form of flow chart as below.

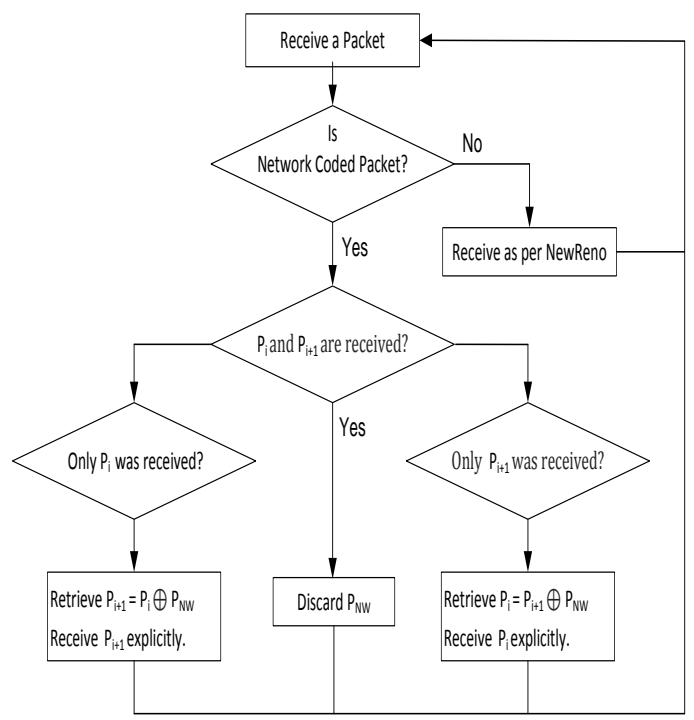

Figure 4. Receiver Side Flowchart

\section{SIMULATION}

Simulation is performed with NS 2.35. Two TCP connections carrying FTP Traffic are used and analysed independently. Various parameters are listed below:

\subsection{Wireless Multihop Networks}

Wireless multihop networks are designed with various parameters. They are broadly categorized based on poor network based or congested network based. In each of these two categories, further categories are based on number of nodes $(100,200,300,400$ and 500) and number of hopes between a TCP connection(5,10 and 15). So total 30 wireless multihop networks can be designed. All these 30 scenarios are tested over TCP NewReno(Without Network Coding) and TCPNC (TCP with Network Coding). So total 60 scenarios have been implemented and various parameters are calculated as below.

\subsection{Average Throughput in KBPS}

Table 2. Average Throughput for various scenarios

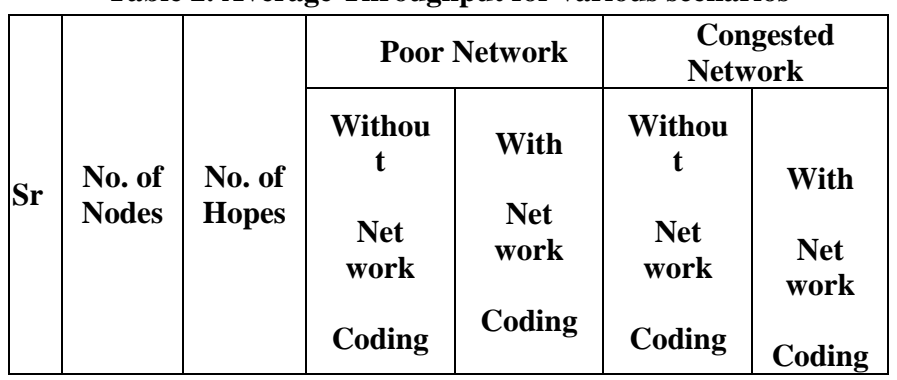

\begin{tabular}{|l|l|c|c|c|c|c|}
\hline & & & & & & \\
\hline 1 & 100 & 5 & 215 & 215 & 184 & 212 \\
\hline 2 & 100 & 10 & 176 & 217 & 213 & 199 \\
\hline 3 & 100 & 20 & 113 & 115 & 133 & 126 \\
\hline 4 & 200 & 5 & 642 & 642 & 647 & 645 \\
\hline 5 & 200 & 10 & 324 & 324 & 277 & 319 \\
\hline 6 & 200 & 20 & 165 & 177 & 207 & 152 \\
\hline 7 & 300 & 5 & 648 & 647 & 646 & 647 \\
\hline 8 & 300 & 10 & 325 & 325 & 318 & 317 \\
\hline 9 & 300 & 20 & 307 & 321 & 315 & 216 \\
\hline 10 & 400 & 5 & 274 & 274 & 238 & 145 \\
\hline 11 & 400 & 10 & 207 & 207 & 232 & 205 \\
\hline 12 & 400 & 20 & 183 & 183 & 124 & 113 \\
\hline 13 & 500 & 5 & 646 & 646 & 645 & 647 \\
\hline 14 & 500 & 10 & 580 & 580 & 582 & 580 \\
\hline 15 & 500 & 20 & 324 & 324 & 310 & 317 \\
\hline
\end{tabular}

4.3 Packet Delivery Ratio in percentage Table 3. Packet Delivery Ratio for various scenarios

\begin{tabular}{|c|c|c|c|c|c|c|}
\hline \multirow[b]{2}{*}{$\mathbf{S r}$} & \multirow[b]{2}{*}{$\begin{array}{l}\text { No. } \\
\text { of } \\
\text { Node } \\
\text { S }\end{array}$} & \multirow[b]{2}{*}{$\begin{array}{l}\text { No } \\
\cdot \\
\text { of } \\
\text { H } \\
\text { op } \\
\text { es }\end{array}$} & \multicolumn{2}{|c|}{ Poor Network } & \multicolumn{2}{|c|}{$\begin{array}{l}\text { Congested } \\
\text { Network }\end{array}$} \\
\hline & & & $\begin{array}{l}\text { With } \\
\text { out } \\
\text { Net } \\
\text { work } \\
\text { Coding }\end{array}$ & $\begin{array}{l}\text { With } \\
\text { Net } \\
\text { work } \\
\text { Coding }\end{array}$ & $\begin{array}{l}\text { With } \\
\text { out } \\
\text { Net } \\
\text { work } \\
\text { Coding }\end{array}$ & $\begin{array}{l}\text { With } \\
\text { Net } \\
\text { work } \\
\text { Coding }\end{array}$ \\
\hline 1 & 100 & 5 & $\begin{array}{c}99.852 \\
3 \%\end{array}$ & $\begin{array}{c}99.890 \\
8 \%\end{array}$ & $\begin{array}{c}99.978 \\
1 \%\end{array}$ & $\begin{array}{c}99.942 \\
9 \%\end{array}$ \\
\hline 2 & 100 & 10 & $\begin{array}{c}99.819 \\
6 \%\end{array}$ & $\begin{array}{c}99.879 \\
0 \%\end{array}$ & $\begin{array}{c}99.949 \\
4 \%\end{array}$ & $\begin{array}{c}99.966 \\
4 \%\end{array}$ \\
\hline 3 & 100 & 20 & $\begin{array}{c}99.883 \\
5 \%\end{array}$ & $\begin{array}{c}99.886 \\
4 \%\end{array}$ & $\begin{array}{c}99.990 \\
0 \%\end{array}$ & $\begin{array}{c}99.917 \\
0 \%\end{array}$ \\
\hline 4 & 200 & 5 & $\begin{array}{c}99.997 \\
8 \%\end{array}$ & $\begin{array}{c}99.997 \\
8 \%\end{array}$ & $\begin{array}{c}99.981 \\
3 \%\end{array}$ & $\begin{array}{c}99.997 \\
9 \%\end{array}$ \\
\hline 5 & 200 & 10 & $\begin{array}{c}99.901 \\
8 \%\end{array}$ & $\begin{array}{c}99.901 \\
8 \%\end{array}$ & $\begin{array}{c}99.980 \\
6 \%\end{array}$ & $\begin{array}{c}99.966 \\
3 \%\end{array}$ \\
\hline 6 & 200 & 20 & $\begin{array}{c}99.938 \\
0 \%\end{array}$ & $\begin{array}{c}100.00 \\
0 \%\end{array}$ & $\begin{array}{c}99.967 \\
6 \%\end{array}$ & $\begin{array}{c}99.911 \\
6 \%\end{array}$ \\
\hline 7 & 300 & 5 & $\begin{array}{c}99.927 \\
3 \%\end{array}$ & $\begin{array}{c}99.927 \\
3 \%\end{array}$ & $\begin{array}{c}99.995 \\
8 \%\end{array}$ & $\begin{array}{c}99.977 \\
2 \%\end{array}$ \\
\hline 8 & 300 & 10 & $\begin{array}{c}99.919 \\
1 \%\end{array}$ & $\begin{array}{c}99.919 \\
1 \%\end{array}$ & $\begin{array}{c}99.953 \\
5 \%\end{array}$ & $\begin{array}{c}99.961 \\
9 \%\end{array}$ \\
\hline 9 & 300 & 20 & $\begin{array}{c}99.972 \\
9 \%\end{array}$ & $\begin{array}{c}99.987 \\
6 \%\end{array}$ & $\begin{array}{c}99.974 \\
3 \%\end{array}$ & $\begin{array}{c}99.987 \\
6 \%\end{array}$ \\
\hline 10 & 400 & 5 & $\begin{array}{c}99.929 \\
5 \%\end{array}$ & $\begin{array}{c}99.929 \\
5 \%\end{array}$ & $\begin{array}{c}99.983 \\
1 \%\end{array}$ & $\begin{array}{c}99.916 \\
8 \%\end{array}$ \\
\hline
\end{tabular}




\begin{tabular}{|c|l|l|c|c|c|c|}
\hline 11 & 400 & 10 & $\begin{array}{c}99.859 \\
8 \%\end{array}$ & $\begin{array}{c}99.859 \\
8 \%\end{array}$ & $\begin{array}{c}99.936 \\
3 \%\end{array}$ & $\begin{array}{c}99.973 \\
8 \%\end{array}$ \\
\hline 12 & 400 & 20 & $\begin{array}{c}99.818 \\
9 \%\end{array}$ & $\begin{array}{c}99.818 \\
9 \%\end{array}$ & $\begin{array}{c}99.913 \\
0 \%\end{array}$ & $\begin{array}{c}99.929 \\
1 \%\end{array}$ \\
\hline 13 & 500 & 5 & $\begin{array}{c}99.929 \\
3 \%\end{array}$ & $\begin{array}{c}99.944 \\
3 \%\end{array}$ & $\begin{array}{c}99.989 \\
6 \%\end{array}$ & $\begin{array}{c}99.977 \\
2 \%\end{array}$ \\
\hline 14 & 500 & 10 & $\begin{array}{c}99.971 \\
3 \%\end{array}$ & $\begin{array}{c}99.971 \\
3 \%\end{array}$ & $\begin{array}{c}99.988 \\
4 \%\end{array}$ & $\begin{array}{c}99.981 \\
5 \%\end{array}$ \\
\hline 15 & 500 & 20 & $\begin{array}{c}99.918 \\
7 \%\end{array}$ & $\begin{array}{c}99.918 \\
7 \%\end{array}$ & $\begin{array}{c}99.974 \\
0 \%\end{array}$ & $\begin{array}{c}99.995 \\
8 \%\end{array}$ \\
\hline
\end{tabular}

\subsection{Instantaneous Throughput}

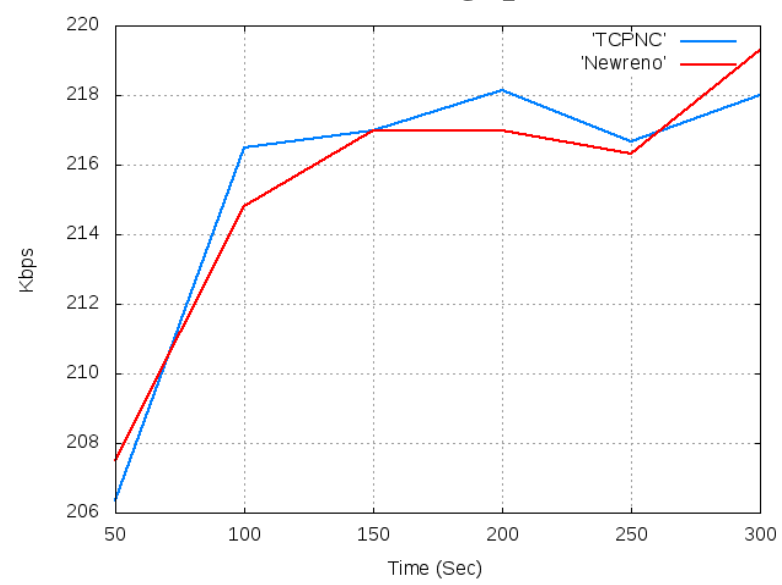

Figure 5. Comparison of TCPNC with New Reno for Poor Network

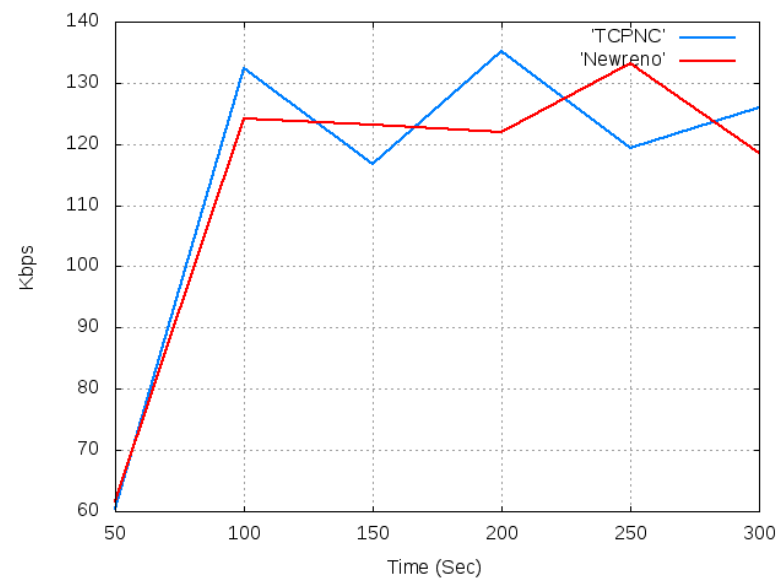

Figure 6. Comparison of TCPNC with New Reno for Congested Network

\section{CONCLUSION}

Wireless multihop networks are becoming more and more popular with reference of the real world implementations. Several categories of wireless multihop networks like adhoc networks, sensor networks are started being widely used. Wireless networks have their own limitations and disadvantage because of wireless communication and inherent broadcast nature. The main issues with wireless networks are they are more vulnerable to transmission problems like attenuation, noise, collision etc. at the same time; they have to compromise with the available bandwidth.
Network coding is a way of improving reliability via increasing redundancy. Several techniques of network coding can be broadly classified as per the layer at which they work. Network coding provides error control without need of retransmission at certain level. At the same time, it helps in reducing bandwidth utilization for some cases.

TCP - Transmission Control Protocol is connection oriented and reliable transport layer protocol. In this thesis, network coding is implemented with TCP's most widely deployed variant named TCP NewReno to form a new TCP variant named TCPNC - TCP with Network Coding.

TCP NewReno and TCPNC are tested over more than 60 scenarios. It has been noticed that TCPNC performs best with poor network. Naturally because of the redundancy of packets, TCPNC performs bit slow with congested network. It has been also noticed that TCPNC performs better upto the wireless multihop network of 300 nodes. Even though a performance is bit slowly with congested network, it is acceptable as network coding is more suitable and required for the networks which are poor in terms of signal strengths, attenuation, random channel loss etc.

\section{FUTURE WORK}

Wireless multihop networks are becoming more and more popular with reference of the real world TCP NewReno and TCPNC are tested over more than 60 scenarios. It has been noticed that TCPNC performs best with poor network. Naturally because of the redundancy of packets, TCPNC performs bit slow with congested network. In future, TCPNC can be modified to identify level of congestion and to act accordingly. TCPNC can be modified to monitor network congestion and activate or deactivate itself on requirement basis.

It has been also noticed that TCPNC performs better upto the wireless multihop network of 300 nodes. TCPNC can be modified to support large number of networks.

As researcher has found that TCPNC performs best with the networks which are poor in terms of signal strengths, attenuation, random channel loss etc., TCPNC can be tested over mobile adhoc networks, wireless sensor networks etc.

Network coding can be implemented at various level of TCP/IP layers. TCPNC is exclusively implemented with TCP protocol at transport layer. In future, TCPNC can be used with other network coding techniques which are implemented at network, data link, physical layers. TCPNC can be tested with real time application based traffic or can be deployed with a real network for efficient analysis.

\section{REFERENCES}

[1] Christina Fragouli, Emina Soljanin, "Network Coding Applications",Foundations and Trends in Networking

[2] Christina Fragouli, Jean-Yves Le Boudec, "Network Coding: An Instant Primer" ACM SIGCOMM Computer Communication Review, Volume 36, January 2006.

[3] Hao Kun, Jin Zhigang, Wang Ying, "Partial Network Coding for Wireless Opportunistic Routing", IET Conference Publications 2009.

[4] Shengli Fu, Kejie Lu, Yi Qian, and Murali Varanasi, "Cooperative Network Coding for Wireless Ad-Hoc Networks", IEEE GLOBECOM 2007 proceedings.

[5] Wei Chen, Khaled B. Letaief, and Zhigang Cao,"Opportunistic Network Coding for Wireless 
Networks", ICC 2007 proceedings [7] Lu Lu, Ming Xiao, Mikael Skoglund, Lars Rasmussen, Gang Wu, Shaoqian Li, "Efficient Network Coding for Wireless Broadcasting", WCNC 2010 proceedings

[6] Shuo-Yen Robert Li, Raymond W. Yeung , "Linear Network Coding", IEEE Transactions on Information Theory, VOL. 49, NO. 2, February 2003

[7] Rodica Stoian, Lucian Andrei Perisoara, Radu Stoica, "Random Network Coding for Wireless Ad-Hoc Networks", Wireless Communications and Networking Conference WCNC IEE 2010

[8] Tracey Ho, Muriel M'edard, Ralf Koetter, David R. Karger, Michelle Effros, Jun Shi and Ben Leong, "A random linear network coding approach to Multicast", IEEE Trans. Information Theory, Oct 2006
[9] Philip Chou, Yunnan Wu, Kamal Jain, "Practical Network Coding", in Proc. Allerton Conf. Commun., 2003

[10] Mohammed Halloush, Hayder Radha, "Network Coding with Multigeneration Mixing", ICC 2008 proceedings

[11] Mohammed Halloush, Hayder Radha, "A Case Study of: Sender Transmission Reliability and Complexity Using Network Coding with Multi-generation Mixing", ICC 2009 proceedings

[12] Mohammed Halloush, Hayder Radha, "Network Coding with Multi- Generation Mixing: Analysis and Applications for Video Communication", ICC 2008 proceedings 